\title{
METODOLOGÍA GENERAL PARA CORRELACIONAR SAGS CON EVENTOS AMBIENTALES U OPERATIVOS DEL SISTEMA DE DISTRIBUCIÓN
}

\author{
Alejandro Castillo Guasca \\ Tecnólogo Electricista \\ Deltec S.A. \\ acastillog@correo.udistrital.edu.co \\ Bogotá, Colombia \\ Edwin Giovanny Cuenca Moreno \\ Tecnólogo Electricista \\ CODENSA S.A. \\ egcuencam@correo.udistrital.edu.co \\ Bogotá, Colombia
}

\section{Yaqueline Garzón Rodríguez}

Magister en Ciencias de la Información y las Comunicaciones

Docente planta de la Universidad Distrital Francisco José de Caldas ygarzonr@udistrital.edu.co

Bogotá, Colombia

Tipo: Artículo de reflexión

Fecha de Recepción: Enero 11 de 2013 Fecha de Aceptación: Marzo 9 de 2013

\section{GENERAL METHODOLOGY TO CORRELATE SAGS WITH ENVIRONMENTAL OR OPERATING EVENTS OF THE DISTRIBUTION SYSTEM}

\begin{abstract}
It is important to know which are the causes of sags occurrency in busbars distribution, due to the fact that involvement of industrial customers is naturally relevant. For this reason we raised a methodology in order to find the correlation between sags and environmental or operative events of the distribution system. As a sample test, the methodology was applied in three specific areas located in different parts of the city. To develop this methodology, data correlation was implemented through dynamic tables and programming in Access, which showed what kind of events are those that have a greater impact on customers. According to the results obtained, some recommendations are suggested in order to mitigate these types of events in the distribution system.
\end{abstract}

Key words: system events, voltage subsidence, sag.

\section{RESUMEN}

Es de gran importancia conocer cuáles son las causas para que se presenten sags en las barrajes de distribución, debido a que la afectación sobre los clientes industriales es de carácter relevante. Por esta razón se planteó una metodología para buscar la correlación existente entre sags y los eventos ambientales u operativos del sistema de distribución. A manera de ejemplo, se aplicó en tres subestaciones, las cuales se encuentran ubicadas en diferentes sectores de la ciudad. Para el desarrollo de esta metodología, se realizó la correlación de los datos mediante la implementación de tablas dinámicas y programación en Access, donde se evidenció qué tipo de eventos son los que tienen un mayor impacto sobre los clientes. De acuerdo a los resultados obtenidos se sugiere algunas recomendaciones para mitigar este tipo de eventos en el sistema de distribución.

Palabras claves: eventos del sistema, hundimientos de tensión, sags. 


\section{INTRODUCCIÓN}

Puesto que se conocen pocos estudios que proporcionen información acerca de la relación de sags con eventos del sistema de tipo ambiental u operativo como es el caso [1], en el cual desarrolla un análisis para verificar el origen de los eventos de tensión en una zona de distribución de tipo rural, es fundamental identificar sus causas con el fin de mitigar los efectos que ocasionan este tipo de fluctuaciones de tensión.

Los principales afectados son los clientes industriales, debido a que la existencia de este tipo de eventos ocasiona irregularidades en el funcionamiento normal de su producción, pérdida de materia prima y paros no programados que finalmente se traducen en sobrecostos.

Las industrias modernas utilizan equipos que son altamente sensibles a las fluctuaciones de tensión debido a que el desarrollo tecnológico y de automatización con los que estos están compuestos son en su gran mayoría componentes electrónicos, que dan origen a fallas y salidas del sistema de producción de varios minutos y hasta horas. Por esta razón es pertinente identificar la correlación existente entre los sags y los eventos ambientales u operativos del sistema de distribución de energía eléctrica de la ciudad, determinando una metodología donde se evidencie claramente la clasificación detallada de fallas y de causas concernientes a los eventos de corta duración sags.

En lo referente a la parte de normatividad Colombiana, la Comisión Reguladora de Energía y Gas (CREG) emitió la resolución 024 de 2005 [2], donde sólo se definen los lineamientos que debe cumplir el operador de red respecto a la tensión de estado estable, dejando de lado el tema de los eventos con duración inferior a un minuto. Para estos eventos no existe a la fecha ninguna penalización ni límites, aunque si se estableció por parte de la CREG, una política de autocontrol manifestada en la CREG 016-2007 [3], donde se establecen pautas a las empresas operadoras de red en el almacenamiento, plan de sistema de medición-registro y el mismo envío de información, de los respectivos sistemas de medición y registro de calidad de potencia.

\section{DEFINICIONES ASOCIADAS A LOS HUNDIMIENTOS DE TENSIÓN}

Debido a que la calidad de energía eléctrica no sólo se fundamenta en la continuidad del servicio, si no también en la presencia de perturbaciones transitorias o momentáneas en la red de distribución. En la actualidad han tomando gran importancia las distorsiones en la onda de tensión, las cuales se asocian a flickers, swells, y sags, estos últimos pueden ocasionar verdaderos "desastres" para los usuarios que van desde la pérdida de información hasta tener que reinicializar todo un proceso industrial con una posible pérdida de materia prima. Se puede profundizar en lo referente a los efectos de corta duración en el capítulo 5 de [4].

Estos eventos son usualmente asociados con fallas remotas al sistema de potencia, por esta razón se propone una metodología de correlación entre los eventos del sistema y los hundimientos de tensión en la cual se deben tener en cuenta las siguientes definiciones básicas.

\subsection{Definición norma NTC 5001}

"Los hundimientos de tensión (sags) son reducciones del valor eficaz de la tensión por debajo del $90 \%$ y por encima del $10 \%$ de la tensión declarada, seguida por un retorno a un valor más alto que el $90 \%$ de la tensión declarada, en un tiempo que varía desde los $8.33 \mathrm{~ms}$ (medio ciclo a $60 \mathrm{~Hz}$ ) hasta 1 minuto. Los hundimientos de tensión son caracterizados por su duración y por la magnitud de la caída (ver figura 1).

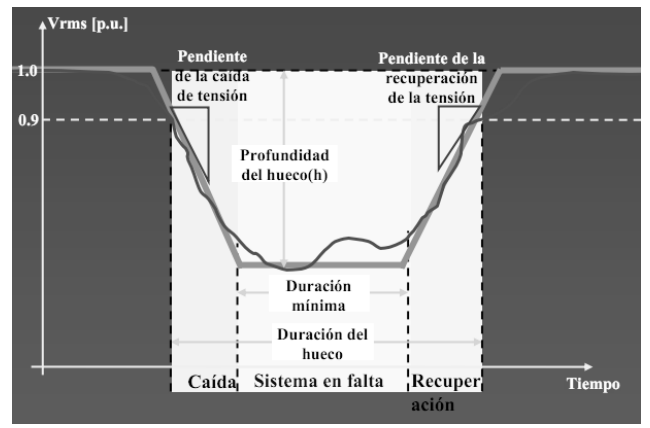

Figura 1. Características hundimiento de tensión [5]. 


\subsubsection{Causas}

Son generalmente producidas por corrientes elevadas, generadas por cortocircuitos, conexión u operación de cargas que exijan una elevada demanda de potencia. Los hundimientos son impredecibles y de comportamiento aleatorio. Este tipo de perturbaciones es una de las que está causando mayor impacto en los equipos instalados en el sistema eléctrico, y dependen en gran medida de la configuración del sistema, del punto de medida, de las condiciones operativas y del mantenimiento entre otros.

Dentro de sus características se tiene:

- Fallas en la fuente de alimentación del sistema de potencia, del sistema eléctrico propio o de vecinos.

- Arranque de motores.

- Cortocircuitos en el sistema de alimentación originados por fenómenos naturales como: arboles, rayos, acciones de terceros, entre otros.

\subsubsection{Efectos}

- Paradas de procesos.

- Reducciones de luz visible de algunos dispositivos de iluminación.

- Caída de sistemas telefónicos.

- Disparo de protecciones.

- Funcionamiento defectuoso de equipos.

- Cambios de velocidad maquinas de inducción.

- Reducción de la potencia de salida en bancos de condensadores.

\subsection{Metodología de evaluación}

Los hundimientos de tensión, se determinan de acuerdo a su duración y magnitud. La duración se define como el tiempo medido desde el momento en que el valor eficaz de la tensión se encuentra por debajo del umbral, hasta cuando retorna por encima de este. La magnitud, está dada por la máxima desviación del valor eficaz de la tensión de la fase más afectada.

Tiempo Inicial: Momento en que alguna de las fases este por debajo del umbral.

Tiempo Final: Cuando todas las fases alcanzan el umbral [6].

\subsection{Eventos de falla del sistema}

Es el registro de interrupciones originadas por fallas o mantenimientos preventivos $\mathrm{y} / \mathrm{o}$ correctivos efectuados sobre la infraestructura eléctrica de determinado sector del Sistema de Distribución Local (SDL).

El sistema de distribución de energía eléctrica está expuesto a eventos de fallas del sistema que afectan su normal funcionamiento. Se denominan eventos ambientales a aquellos que se encuentran directamente relacionados con el medio ambiente como las lluvias, descargas atmosféricas, viento y la interferencia de ramas de los arboles sobre las líneas de distribución entre otros. Por otra parte se tienen los eventos de tipo operativo que se producen por errores del operador de red o por la avería de algún elemento del circuito.

\section{METODOLOGÍA PROPUESTA PARA LA CORRELACIÓN DE HUNDIMIENTOS DE TENSIÓN CON EVENTOS DE FALLAS DEL SISTEMA}

Esta metodología consiste en encontrar la correlación entre los eventos del sistema (ambientales - operativos) y los hundimientos de tensión. El desarrollo de esta, permite determinar de manera general cuáles son los eventos que se presentan con una mayor frecuencia y a su vez identificar cuáles de estos tiene una mayor afectación en los clientes. La metodología está conformada en cuatro etapas, las cuales se dentifican en la figura 2. 


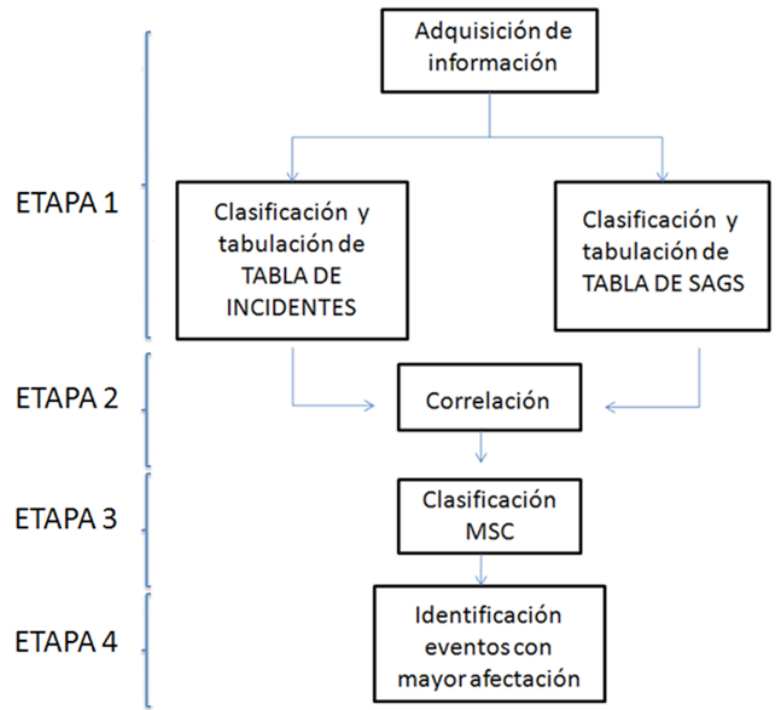

Figura 2. Diagrama metodología. Fuente: Elaboración propia.

\subsection{Etapa 1: Adquisición y clasificación de datos}

En primer lugar se debe obtener la información de eventos del sistema y de hundimientos de tensión.

\subsubsection{Eventos del sistema}

En el sistema de distribución de energía eléctrica se presentan diferentes tipos de eventos, para registrarlos se deben tener en cuentan las siguientes características:

- Mes .

- Fecha de inicio y de fin de la incidencia (dd/mm/aaaa).

- Hora de inicio y de fin de la incidencia (hh:mm), formato 12 horas.

- Subestación y barraje asociados con el evento de falla.

- Causa relacionada con el evento.

Según lo estipulado en el parágrafo 11.2 "Calidad del servicio en el sistema de distribución local (SDL)" en [7], se establece la clasificación de las interrupciones, independientemente de su duración o del número de fases de cada circuito o transformador afectadas, adicionalmente existe un clasificación previa en [8], de los tipos de causas de las interrupciones que deben ser reportadas al Sistema Único de Información (SUI). Para el registro de incidencias de los eventos del sistema en esta metodología se proponen cuatro grupos de causas como complemento de lo establecido en [8], las causas se clasifican en causa externa, causa externa ambiental, causa operativa y causa no determinada relacionadas en la tabla 1.

Tabla 1. Clasificación eventos del sistema. Fuente: Elaboración propia

\begin{tabular}{|c|c|}
\hline CAUSA EXTERNA & \\
\hline Descripción de Causa & Tipo \\
\hline Línea rota & No programada \\
\hline Defecto en conector & No programada \\
\hline Defecto en poste & No programada \\
\hline Acción de Terceros & No programada \\
\hline Otros Objetos sobre la red & No programada \\
\hline Defecto en cortocircuito & No programada \\
\hline Defecto en pararrayos & No programada \\
\hline Defecto en transformador & No programada \\
\hline Defecto en aisladores & No programada \\
\hline Defecto en cruceta & No programada \\
\hline Defecto en seccionador & No programada \\
\hline Defecto en interruptor & No programada \\
\hline Defecto en reconectador & No programada \\
\hline Defecto en condensador & No programada \\
\hline Defecto en otros equipos & No programada \\
\hline Línea suelta & No programada \\
\hline Seguridad Ciudadana & Terceros \\
\hline $\begin{array}{l}\text { Activo de nivel de tensión } 1 \text { de } \\
\text { propiedad del usuarios }\end{array}$ & Terceros \\
\hline \multicolumn{2}{|c|}{ CAUSA EXTERNA AMBIENTAL } \\
\hline Descripción de Causa & Tipo \\
\hline Árbol o rama sobre línea & No programada \\
\hline Condiciones Atmosféricas & No programada \\
\hline \multicolumn{2}{|c|}{ CAUSA OPERATIVA } \\
\hline Descripción de Causa & Tipo \\
\hline Sobrecarga en circuito & No programada \\
\hline Acercamiento de barra & No programada \\
\hline Error involuntario & No programada \\
\hline
\end{tabular}




\begin{tabular}{|c|c|}
\hline Variación de tensión & No programada \\
\hline Falla en transformador & No programada \\
\hline Variación de frecuencia & No programada \\
\hline Error de operación & No programada \\
\hline $\begin{array}{c}\text { Incumplimiento en contrato de } \\
\text { servicios públicos }\end{array}$ & Programada \\
\hline Trabajos de poda de árboles & Programada \\
\hline Mantenimiento en redes & Programada \\
\hline Solicitud de particulares & Programada \\
\hline Remodelación de la red & Programada \\
\hline $\begin{array}{c}\text { Programa anual de } \\
\text { remodelación o reposición }\end{array}$ & Programada \\
\hline Interrupción en STR & Terceros \\
\hline Interrupción en STN & Terceros \\
\hline Racionamiento en el SIN & Terceros \\
\hline Indisponibilidad de activos STR & Terceros \\
\hline Indisponibilidad de activos STN & Terceros \\
\hline \multicolumn{2}{|c|}{ CAUSA NO DETERMINADA } \\
\hline Descripción de Causa & Tipo \\
\hline Causa Desconocida & No programada \\
\hline
\end{tabular}

\subsubsection{Hundimientos de tensión}

Los equipos analizadores de red deben registrar hundimientos de tensión asociados a cada uno de los barrajes de la subestación. La característica de este tipo de registro debe ser:

- Mes.

- Fecha de registro del hundimiento (dd/mm/aaaa).

- Hora de registro del hundimiento (hh:mm), formato 12 horas.

- Subestación y barraje asociada al hundimiento de tensión.

- Duración del hundimiento de tensión.

- Magnitud del hundimiento de tensión, para cada una de las fases.

\subsection{Etapa 2: Correlación de datos}

En la segunda etapa se correlacionan lo eventos de fallas del sistema con los hundimientos de tensión, para ello es indispensable tener en cuenta que exista una relación de fecha y hora con un intervalo de tiempo no mayor a $3 \mathrm{mi}-$ nutos (tiempo promedio de diferencia entre los dos registros de cada base de datos).

El procedimiento para dicha correlación se puede realizar mediante Excel con la implementación de tablas dinámicas, Access o mediante un software de minería de datos.

\subsection{Etapa 3: Clasificación de hundimientos de tensión según MSC}

En esta etapa se realiza una clasificación de todos los hundimientos de tensión registrados por los equipos analizadores de red, para cada uno de los barrajes. La clasificación se hará a partir de la severidad del hundimiento según tres regiones planteadas por la Universidad Nacional en [9], que se pueden identificar en la figura 3.

- Región de sags tipo M (moderados) en la cual, a pesar de que ocurran sags, se tiene una probabilidad muy baja de que algún equipo vea afectada su operación o condición de funcionamiento.

- Región de sags tipo S (severos) en la cual los sags pueden sacar de funcionamiento a unos equipos pero a otros no.

- Región de sags tipo C (críticos) en la cual un sag saca de funcionamiento a cualquier equipo del sistema.

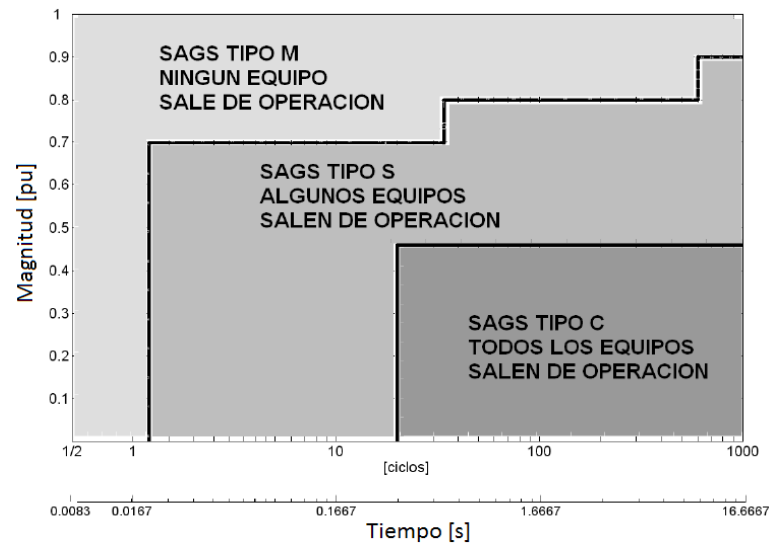

Figura 3. Clasificación M,S,C. [9].

Se aplica "análisis de Pareto"; para identificar dentro de muchos eventos del sistema cuales son los que presentan una mayor concurrencia 
con el fin de determinar qué tipo de severidad ocasionan a los usuarios, en busca de disminuir la presencia de sags en las barras de distribución.

\section{APLICACIÓN DE LA METODOLOGÍA EN TRES ZONAS DE LA CIUDAD DE BOGOTÁ}

Se empleo esta metodología en tres subestaciones ubicadas en distintas zonas de la ciudad, en busca de evaluar cual de ellas presenta una mayor presencia de hundimientos de tensión, como se ven a continuación:

- Zona sur (subestación A).

- Zona centro (subestación B).

- Zona norte (subestación C).

El procedimiento que se llevó a cabo para la aplicación de la metodología sugerida en estas tres subestaciones esta comprendido entre (Enero - Agosto del 2010) y se indica por las siguientes etapas.

\subsection{Etapa 1: Adquisición y clasificación de la información}

En esta primera etapa se obtuvieron las bases de datos correspondientes de eventos de falla del sistema en los niveles de tensión $(11.4 \mathrm{kV}-34.5 \mathrm{kV})$, como también la base de datos de archivos ET (hundimientos de tensión registrados por el analizador de red NEXUS) según lo estipulado en [2] y [10], cumpliendo cada una de ellas con las características delimitadas previamente en la metodología propuesta.

\subsubsection{Base de datos de Incidencias (Eventos del Sistema)}

- Mes (Enero-Agosto 2010).

- Fecha de inicio y de fin de la incidencia (dd/mm/aaaa) (Enero-Agosto 2010).

- Hora de inicio y de fin de la incidencia (hh:mm), formato 12 horas (Enero-Agosto
2010).

- Subestación y barraje asociados a la afectación.

- Causa relacionada con el evento.

A esta base de datos corresponde un total de 374 eventos de fallas del sistema para las tres subestaciones, las cuales comprenden un delta de tiempo entre la fecha de inicio y fecha de fin por incidente, a su vez cada uno constituye en su contenido las causas específicas a tener en cuenta para próximas recomendaciones. A continuación se presenta el indicador de incidencias por subestación, en la figura 4.

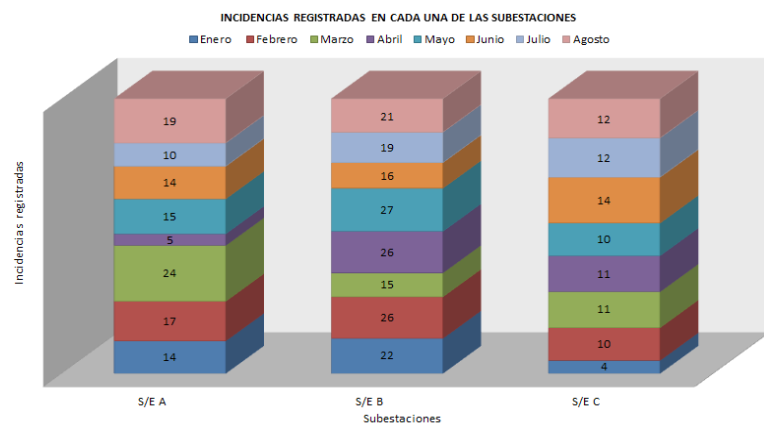

Figura 4. Indicador de incidencias por subestación. Fuente: Elaboración propia.

Se identifica en la grafica de eventos de falla del sistema que en el mes de Mayo en la subestación $\mathrm{C}$, se registraron el mayor número de incidencias las cuales se encuentran relacionadas con lluvias, descargas atmosféricas y degradación de material.

Para la tabla 2 se identificaron dos tipos de eventos que no se encontraban en la clasificación dada por la CREG en [8], es el caso de la presencia de animales en las líneas de distribución que corresponde a un tipo de causa externa y degradación del material que están relacionados con el tipo de causa operativa. A continuación se muestran los tipos de eventos del sistema que se registraron y clasificaron a partir de la tabla 1 , durante el periodo de análisis. 
Tabla 2. Causas eventos de falla del sistema.

Fuente: Elaboración propia

\begin{tabular}{|c|c|c|}
\hline CAUSA EXTERNA & CAUSA EXTERNA AMBIENTAL & CAUSA OPERATIVA \\
\hline Animales & Árbol o rama sobre línea & Degradación de material \\
\hline $\begin{array}{c}\text { Cometas - Otros objetos sobre la } \\
\text { red }\end{array}$ & \multirow{2}{*}{$\begin{array}{l}\text { Descargas atmosféricas (rayos) - } \\
\text { Condiciones Atmosféricas }\end{array}$} & $\begin{array}{c}\text { Error operador de terreno - Error de } \\
\text { operación }\end{array}$ \\
\hline $\begin{array}{c}\text { Equipos de obras civiles - Acciones } \\
\text { de terceros }\end{array}$ & & $\begin{array}{c}\text { Mantenimiento preventivo - Mantenimiento } \\
\text { en redes }\end{array}$ \\
\hline $\begin{array}{l}\text { Instalación e interferencia cliente - } \\
\text { Acciones de terceros }\end{array}$ & \multirow{2}{*}{$\begin{array}{l}\text { Lluvia - Condiciones } \\
\text { Atmosféricas }\end{array}$} & $\begin{array}{l}\text { Falla en Baja Tensión - Activo de nivel de } \\
\text { tensión } 1 \text { de propiedad del usuarios }\end{array}$ \\
\hline Vandalismo - Acciones de terceros & & Fallo equipo de control - Error de operación \\
\hline $\begin{array}{l}\text { Personas Accidental - Acciones de } \\
\text { terceros }\end{array}$ & \multirow{2}{*}{$\begin{array}{l}\text { Viento - Condiciones } \\
\text { Atmosféricas }\end{array}$} & \multirow{2}{*}{ Sobrecargas - Error de operación } \\
\hline $\begin{array}{l}\text { Vehículos Accidental - Acciones de } \\
\text { terceros }\end{array}$ & & \\
\hline
\end{tabular}

\subsubsection{Base de datos Archivos ET (hundimientos} de tensión registrados por el analizador de red NEXUS, en los niveles de tensión $11.4 \mathrm{kV}$ y $34.5 \mathrm{kV}$ )

- Mes (Enero-Agosto 2010).

- Fecha de registro del hundimiento (dd/ $\mathrm{mm} / \mathrm{aaaa}$ ) (Enero-Agosto 2010).

- Hora de registro del hundimiento (hh:mm), formato 12 horas (Enero-Agosto 2010).

- Subestación y barraje asociada al hundimiento.

- Duración del hundimiento de tensión.

- Magnitud del hundimiento de tensión.

A esta base de datos corresponde un total de 18,363 registros de hundimientos de tensión que se muestran en la figura 5.

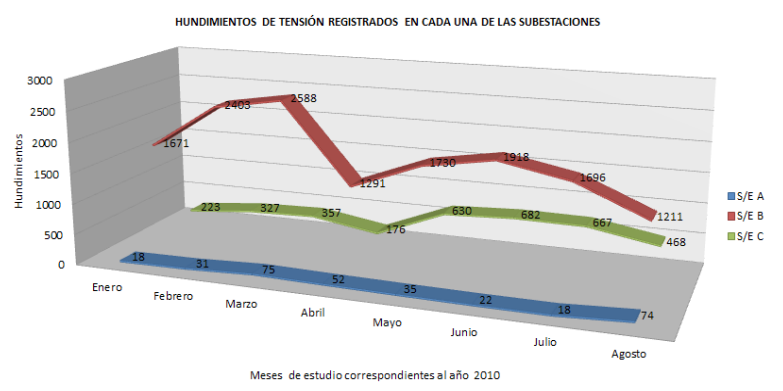

Figura 5. Cantidad de eventos sags presentados. Fuente: Elaboración propia.
A partir de las subestaciones de estudio y de los registros suministrados por el analizador de red, se evidencia que la zona centro presenta un mayor número de hundimientos de tensión, específicamente en el mes de marzo donde se encuentra el punto critico de 2588 hundimientos de tensión.

\subsection{Etapa 2: Correlación de los eventos del sistema con los hundimientos de tensión}

El procedimiento que se llevó a cabo para la aplicación de esta segunda etapa en las tres subestaciones, se realizó mediante Excel de forma manual con la implementación de tablas dinámicas, pero a su vez se desarrollo un código en Access, con el fin de obtener una correlación más efectiva.

A continuación en la figura 6, se muestra las fases implementadas para correlacionar en Access las bases correspondientes a "Hundimientos de Tensión" con las "Incidencias del Sistema". 


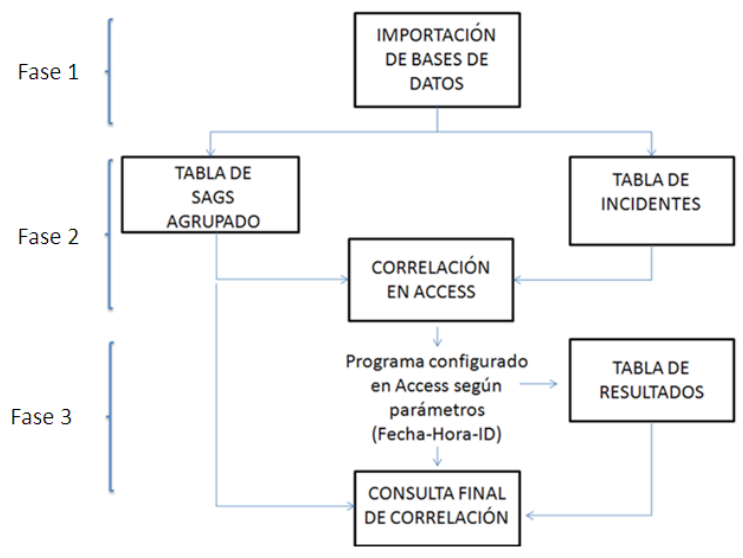

Figura 6. Metodología desarrollada. Fuente: Elaboración propia.

\subsubsection{Fase 1: Importación de tablas en Access}

Como primera medida se importan en Access las tablas de Excel, en primer lugar la tabla que nos genera el Sistema de información América (SDA) "Maestro de incidencias" y la tabla que nos reporta el analizador de red Nexus (archivos ET - tabla de hundimientos de tensión), mencionando que estos últimos han sido consolidados anteriormente para las tres subestaciones de estudio.

\subsubsection{Fase 2: Agrupación de archivos ET}

En esta etapa se agrupa la tabla de los archivos ET, según las características necesarias para la correlación, en este caso se agrupan (Ítem-Fecha-Hora-Subestación-Barra-Duración de Hundimientos de Tensión).

\subsubsection{Fase 3: Elaboración de consultas}

Para esta etapa se crea una consulta de datos anexados, la cual realiza la correlación conservando el ID de la tabla de los archivos ET, mediante los criterios (ID-Fecha-Hora) y los indicadores de la tabla de Incidentes (Subestación-Fecha Inicio - Hora Inicio - Fecha Fin Hora Fin - Causa_SUI - Observaciones) respectivamente, haciendo énfasis en que los primeros se anexan en el programa diseñado en Access. Como parámetros base, para garantizar una correlación donde se vean enlazadas las causas debidas a cada uno de los hundimientos de tensión generados para el periodo asignado.

A partir de la base de datos Archivos ET y ayuda de las tablas dinámicas se identifica la relación de fecha y hora entre ambas bases de datos, conllevando a que la fecha y hora de cada uno de los hundimientos de tensión este comprendido en el delta de tiempo de la base de datos de Incidencias y a su vez el mismo no sea mayor a 3 minutos.

Para observar detalladamente la evidencia de causas más frecuentes por las cuales una zona tiene mayor nivel de severidad de Sags que otra, se presentan a continuación los siguientes resultados:

De 18,363 datos ET de las 3 subestaciones, que fueron registrados por cada uno de los analizadores de red instalados en los barrajes de las subestaciones, 832 correlacionan y cumplen al estar en el rango de fecha y hora de la base de datos de incidencias, relacionando a su vez cada uno de los hundimientos de tensión con la clasificación de eventos del sistema que se reportan al SUI, de acuerdo en lo estipulado en [8]. A continuación en la tabla 3 se muestran los resultados de la clasificación.

Tabla 3. Clasificación Eventos de Fallas del Sistema

Fuente: Elaboración propia

\begin{tabular}{|c|c|c|c|c|}
\hline \multirow{2}{*}{ SUBESTACIÓN } & \multicolumn{4}{|c|}{ CLASIFICACIÓN DE CAUSAS } \\
\cline { 2 - 5 } & EXTERNA & EXTERNA AMBIENTAL & OPERATIVA & NO DETERMINADA \\
\hline A & 31 & 13 & 102 & 12 \\
\hline B & 100 & 74 & 261 & 77 \\
\hline C & 36 & 15 & 97 & 14 \\
\hline Total General & 167 & 102 & 460 & 103 \\
\hline
\end{tabular}




\section{CLASIFICACIÓN EVENTOS DE FALLAS DEL SISTEMA REGISTRADAS}

m EXTERNA = EXTERNA AMBIENTAL =OPERATIVA = NO DETERMINADA

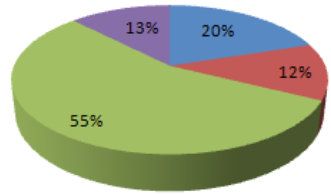

Figura 7. Clasificación eventos de falla del sistema. Fuente: Elaboración propia.

A partir de los resultados obtenidos se observa en la gráfica de la figura 7 , que el mayor número de hundimientos de tensión se deben a causas operativas, se registraron 345 hundimientos de tensión de tipo severo según clasificación MSC. La consecuencia de este tipo de eventos es el de sacar de funcionamiento equipos sensibles a perturbaciones como lo son:

- Computadores personales.

- Sistemas interrumpidos de potencia (UPS).

- Controles de lógica programable (PLC).

- Variadores de frecuencia.

- Motores de corriente directa.

Y cualquier equipo eléctrico del usuario que posea un control electrónico o un sistema de conversión AC-DC o AC-AC.

A continuación, se presenta en cada una de las tablas 4, 5, 6 y 7 un informe detallado de los eventos que se encuentran relacionados a las causas que tuvieron correlación con los hundimientos de tensión, para cada una de las subestaciones respectivamente.

Tabla 4. Registro Causa Externa.

Fuente: Elaboración propia.

\begin{tabular}{|c|c|c|c|}
\hline \multicolumn{4}{|c|}{ CAUSA EXTERNA } \\
\hline$S / E$ & Animales & Total & $\%$ \\
\hline \multirow{4}{*}{ A } & Cometas & 3 & 9,68 \\
\hline & $\begin{array}{c}\text { Instalación e } \\
\text { interferencia cliente }\end{array}$ & 23 & 74,19 \\
\hline & Vandalismo & 1 & 3,23 \\
\hline & Vehículos - accidental & 2 & 6,45 \\
\hline & Total A & 31 & 18,56 \\
\hline
\end{tabular}

\begin{tabular}{|c|c|c|c|}
\hline \multirow[b]{2}{*}{ B } & Animales & 29 & 29,00 \\
\hline & $\begin{array}{c}\text { Instalación e } \\
\text { interferencia cliente }\end{array}$ & 71 & 71,00 \\
\hline \multicolumn{2}{|r|}{ Total B } & 100 & $59,88 \%$ \\
\hline \multirow{6}{*}{$\mathrm{C}$} & Cometas & 14 & 38,89 \\
\hline & Excavadoras & 3 & 8,33 \\
\hline & $\begin{array}{c}\text { Instalación e } \\
\text { interferencia cliente }\end{array}$ & 5 & 13,89 \\
\hline & Personas - accidental & 1 & 2,78 \\
\hline & Vandalismo & 12 & 33,33 \\
\hline & Vehículos - accidental & 1 & 2,78 \\
\hline \multicolumn{2}{|r|}{ Total C } & 36 & 21,56 \\
\hline \multicolumn{2}{|r|}{ Total general } & 167 & 100 \\
\hline
\end{tabular}

Tabla 5. Registro Causa Externa Ambiental.

Fuente: Elaboración propia.

\begin{tabular}{|c|c|c|c|}
\hline \multicolumn{4}{|c|}{ CAUSA EXTERNA AMBIENTAL } \\
\hline S/E & Causa_SUI & Total & $\%$ \\
\hline A & Lluvia & 13 & 100 \\
\hline \multirow{2}{*}{} & Total A & 31 & 12,75 \\
\cline { 2 - 4 } & Arboles & 35 & 47,30 \\
\cline { 2 - 4 } B & $\begin{array}{c}\text { Descarga atmosferica } \\
\text { (rayo) }\end{array}$ & 18 & 24,32 \\
\cline { 2 - 4 } & Lluvia & 6 & 8,11 \\
\cline { 2 - 4 } & Viento & 15 & 20,27 \\
\hline \multirow{2}{*}{ C } & Total B & 74 & $59,88 \%$ \\
\cline { 2 - 4 } & Arboles & 11 & 73,33 \\
\hline \multirow{2}{*}{} & Lluvia & 4 & 26,67 \\
\hline \multicolumn{2}{|c|}{ Total general } & 102 & 100 \\
\hline
\end{tabular}

Tabla 6. Registro Causa Operativa.

Fuente: Elaboración propia

\begin{tabular}{|c|c|c|c|}
\hline \multicolumn{4}{|c|}{ CAUSA EXTERNA } \\
\hline$S / E$ & Animales & Total & $\%$ \\
\hline \multirow{2}{*}{ A } & Degradación material & 29 & 28,43 \\
\hline & Fallo equipo control & 23 & 71,57 \\
\hline \multicolumn{2}{|r|}{ Total A } & 102 & 22,17 \\
\hline
\end{tabular}




\begin{tabular}{|c|c|c|c|}
\hline \multicolumn{4}{|c|}{ CAUSA OPERATIVA } \\
\hline \multirow{4}{*}{ B } & Degradacion material & 196 & 75,10 \\
\hline & Falla en B.T. & 21 & 8,05 \\
\hline & Falta mantenimiento & 42 & 16,09 \\
\hline & Reparto de cargas & 2 & 0,77 \\
\hline \multicolumn{2}{|r|}{ Total B } & 261 & 56,74 \\
\hline \multirow{3}{*}{$\mathrm{C}$} & Degradación material & 89 & 91,75 \\
\hline & $\begin{array}{c}\text { Error operador de } \\
\text { terreno }\end{array}$ & 1 & 1,03 \\
\hline & $\begin{array}{l}\text { Mantenimiento } \\
\text { preventivo }\end{array}$ & 7 & 7,22 \\
\hline \multicolumn{2}{|r|}{ Total C } & 97 & 21,09 \\
\hline \multicolumn{2}{|r|}{ Total general } & 460 & 100 \\
\hline
\end{tabular}

Tabla 7. Registro causa no determinada.

Fuente: Elaboración propia.

\begin{tabular}{|c|c|c|}
\hline \multicolumn{3}{|c|}{ CAUSA NO DETERMINADA } \\
\hline S/E & Total & $\%$ \\
\hline A & 12 & 11,65 \\
\hline B & 77 & 74,76 \\
\hline C & 14 & 13,59 \\
\hline Total general & 103 & 100,00 \\
\hline
\end{tabular}

\subsection{Etapa 3: Clasificación hundimientos de tensión según $(M, S, C)$}

Una vez obtenidos los hundimientos de tensión que correlacionaron con las incidencias, se determinó que tipo de afectación tenía cada uno de ellos según la clasificación MSC, estas se relacionan en las tablas 8, 9, 10 y 11 .

Tabla 8. MSC - Causa externa.

Fuente: Elaboración propia.

\begin{tabular}{|c|c|c|c|c|c|c|c|c|}
\hline \multirow{2}{*}{ SUBESTACIÓN } & \multicolumn{9}{|c|}{ CAUSA EXTERNA } \\
\cline { 2 - 10 } & $\begin{array}{c}\text { TIPO } \\
\text { M }\end{array}$ & $\%$ & $\begin{array}{c}\text { TIPO } \\
\text { S }\end{array}$ & $\%$ & $\begin{array}{c}\text { TIPO } \\
\text { C }\end{array}$ & $\%$ & $\begin{array}{c}\text { NO } \\
\text { DET. }\end{array}$ & $\%$ \\
\hline A & 3 & 33,33 & 17 & 15,18 & 2 & 5,71 & 9 & 81,82 \\
\hline B & 1 & 11,11 & 64 & 57,14 & 33 & 94,29 & 2 & 18,18 \\
\hline C & 5 & 55,56 & 31 & 27,68 & 0 & 0,00 & 0 & 0,00 \\
\hline $\begin{array}{c}\text { TOTAL } \\
\text { GENERAL }\end{array}$ & 9 & 5,39 & 112 & 67,07 & 35 & 20,96 & 11 & 6,59 \\
\hline
\end{tabular}

Tabla 9. MSC - Causa operativa.

Fuente: Elaboración propia.

\begin{tabular}{|c|c|c|c|c|c|c|c|c|}
\hline \multirow{2}{*}{ SUBESTACIÓN } & \multicolumn{8}{|c|}{ CAUSA OPERATIVA } \\
\cline { 2 - 9 } & $\begin{array}{c}\text { TIPO } \\
\text { M }\end{array}$ & $\%$ & $\begin{array}{c}\text { TIPO } \\
\text { S }\end{array}$ & $\%$ & $\begin{array}{c}\text { TIPO } \\
\text { C }\end{array}$ & $\%$ & $\begin{array}{c}\text { NO } \\
\text { DET. }\end{array}$ & $\%$ \\
\hline A & 9 & 8,65 & 86 & 24,93 & 5 & 62,50 & 2 & 66,67 \\
\hline B & 68 & 65,38 & 192 & 55,65 & 0 & 0,00 & 1 & 33,33 \\
\hline C & 27 & 25,96 & 67 & 19,42 & 3 & 37,50 & 0 & 0,00 \\
\hline $\begin{array}{c}\text { TOTAL } \\
\text { GENERAL }\end{array}$ & 104 & 22,61 & 345 & 75,00 & 8 & 1,74 & 3 & 0,65 \\
\hline
\end{tabular}


Tabla 10. MSC - Causa externa ambiental.

Fuente: Elaboración propia.

\begin{tabular}{|c|c|c|c|c|c|c|c|c|}
\hline \multirow{2}{*}{ SUBESTACIÓN } & \multicolumn{8}{|c|}{ CAUSA OPERATIVA } \\
\cline { 2 - 10 } & $\begin{array}{c}\text { TIPO } \\
\text { M }\end{array}$ & $\%$ & $\begin{array}{c}\text { TIPO } \\
\text { S }\end{array}$ & $\%$ & $\begin{array}{c}\text { TIPO } \\
\text { C }\end{array}$ & $\%$ & $\begin{array}{c}\text { NO } \\
\text { DET. }\end{array}$ & $\%$ \\
\hline A & 0 & 0,00 & 13 & 15,66 & 0 & 0,00 & 0 & 0,00 \\
\hline B & 17 & 94,44 & 56 & 67,47 & 1 & 100 & 0 & 0,00 \\
\hline C & 1 & 5,56 & 14 & 16,87 & 0 & 0,00 & 0 & 0,00 \\
\hline $\begin{array}{c}\text { TOTAL } \\
\text { GENERAL }\end{array}$ & 18 & 17,65 & 83 & 81,37 & 1 & 0,98 & 0 & 0,00 \\
\hline
\end{tabular}

Según las tablas proporcionadas por la clasificación MSC, se evidencia claramente que 14 eventos no se encuentran dentro del rango, 2 de ellos porque son mayores a 1000 ciclos y los otros 12 porque la magnitud es menor a la establecida en [6].

\subsection{Etapa 4: Análisis de los resultados - aplicación "Análisis de Pareto"}

El desarrollo de este análisis de Pareto consta de 5 fases:

\section{Fase 1: Definir como clasificar los datos.}

Para la clasificación de los eventos del sistema, se tendrá en cuenta, la presentada en [8]. Se anexaron las causas presencia de animales, de- gradación del material y falta de mantenimiento debido a que no se encontraban dentro de esta clasificación.

Fase 2: Elegir el periodo de observación del fenómeno.

Como se estipulo previamente, comprende desde 01 de Enero de 2010 hasta 30 de agosto de 2010.

\section{Fase 3: Obtener los datos y ordenarlos.}

Los datos que se obtuvieron luego de hacer la correlación en Access entre los eventos del sistema y los sags registrados en las barras de distribución, se relacionan en la tabla 12 . 
Tabla 12. Registros de Eventos de Fallas del Sistema.

Fuente: Elaboración propia.

\begin{tabular}{|c|c|c|c|c|}
\hline ASIGNACIÓN & MAESTRO DE INCIDENCIAS & EVENTOS DEL SISTEMA & № DE EVENTOS & {$[\%]$} \\
\hline \multirow{2}{*}{ A } & Degradación de material & \multirow{2}{*}{ Degradación del material } & \multirow{2}{*}{356} & \multirow{2}{*}{42,79} \\
\hline & Falta de mantenimiento & & & \\
\hline $\mathrm{B}$ & No determinada & Causa desconocida & 103 & 12,38 \\
\hline \multirow{2}{*}{$\mathrm{C}$} & Falla en BT & \multirow{2}{*}{$\begin{array}{l}\text { Activo de nivel de tensión } \\
1 \text { usuario }\end{array}$} & \multirow{2}{*}{98} & \multirow{2}{*}{11,78} \\
\hline & Instalación e interferencia cliente & & & \\
\hline \multirow{2}{*}{$\mathrm{D}$} & Fallo equipo de control & \multirow{2}{*}{ Error de operación } & \multirow{2}{*}{75} & \multirow{2}{*}{9,01} \\
\hline & Reparto de cargas & & & \\
\hline \multirow{3}{*}{$\mathrm{E}$} & Descarga atmosférica & \multirow{2}{*}{ Condiciones atmosféricas } & \multirow{2}{*}{56} & \multirow{2}{*}{6,73} \\
\hline & Lluvia & & & \\
\hline & Viento & & & \\
\hline $\mathrm{F}$ & Arboles & Árbol o rama sobre línea & 46 & 5,53 \\
\hline $\mathrm{G}$ & Cometas & Otros Objetos sobre la red & 37 & 4,45 \\
\hline $\mathrm{H}$ & Animales & Presencia de animales & 32 & 3,85 \\
\hline \multirow{4}{*}{ I } & Vandalismo & \multirow{4}{*}{ Acciones de terceros } & \multirow{4}{*}{21} & \multirow{4}{*}{2,52} \\
\hline & Excavadoras & & & \\
\hline & Personas accidental & & & \\
\hline & Vehículos & & & \\
\hline $\mathrm{J}$ & Mantenimiento preventivo & Mantenimiento en redes & 7 & $0,84 \%$ \\
\hline $\mathrm{K}$ & Error operador de red & Error Involuntario & 1 & $0,12 \%$ \\
\hline \multicolumn{3}{|c|}{ TOTAL } & 832 & 100 \\
\hline
\end{tabular}

\section{Fase 4: Informaciones básicas del estudio.}

En la figura 8, se relaciona los eventos del sistema junto con su recurrencia.

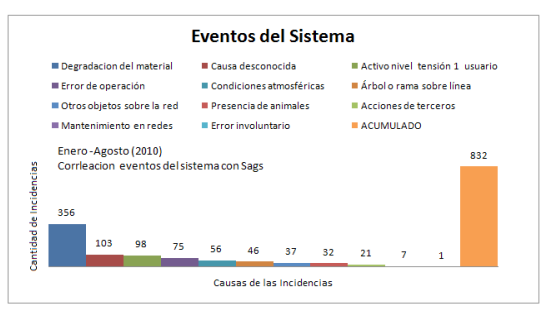

Figura 8. Información eventos de fallas del sistema. Fuente: Elaboración propia.

Fase 5: Buscando el "break point" ó punto de quiebre, que separa los pocos vitales de los muchos triviales.

El análisis de Pareto indica que el $80 \%$ corres- ponde a la zona dudosa donde se encuentran las causas que tiene mayor afectación sobre el problema y el 20\% corresponde a las afectaciones triviales, bajo esta información se obtiene el diagrama de Pareto en la figura 9.

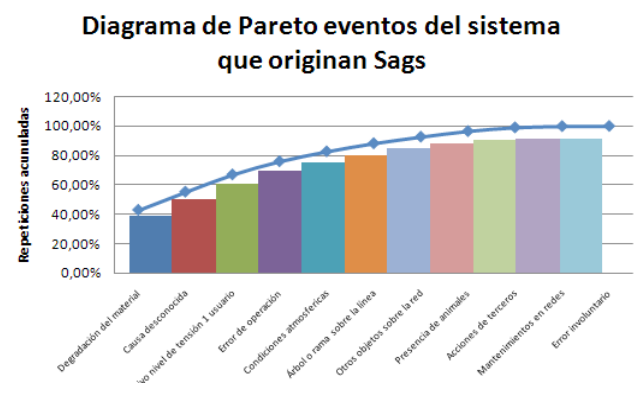

Figura 9. Break point/punto de quiebre . Fuente: Elaboración propia.

A partir de los resultados obtenidos en el análisis, para que Codensa S.A ESP obtenga una dis- 
minución aproximada al $82,17 \%$ en la presencia de sags en las barras de distribución, debe concentrarse en estos 5 eventos del sistema.

- Degradación del material.

- Causa desconocida.

- Activo nivel 1 del usuario.

- Error de operación.

- Condiciones atmosféricas.

El resto de eventos del sistema corresponde al $17,31 \%$, que se clasifican como eventos triviales.

- Árbol o rama sobre la línea.

- Otros objetos sobre la red.

- Presencia de animales.

Según el concepto de análisis de Pareto, podemos decir que al atacar el $45,5 \%$ de los eventos del sistema se resolvería el $82,17 \%$ del problema de presencia de sags en las barras de distribución y el $54,5 \%$ de las causas solo resuelve el $17,31 \%$ del problema.

\section{RECOMENDACIONES A PARTIR DE LOS RESULTADOS}

Debido a que los hundimientos de tensión no se pueden eliminar en su totalidad pero si mitigar, se sugiere a Codensa S.A ESP las siguientes recomendaciones.

Según el periodo de estudio el $80 \%$ de los hundimientos de tensión de tipo crítico se deben a causas externas como lo son: objetos sobre la red, presencia de animales y acciones de terceros, por ello es conveniente realizar mantenimientos preventivos e inspecciones técnicas sobre las redes de distribución de cada una de las subestaciones.

Limpieza de aisladores, inspección técnica de los circuitos de distribución correspondientes a la Subestación B, ya que en el periodo de análisis comprendido entre Enero y Agosto de 2010, se evidencia un índice significativo correspondiente a 261 eventos, relacionados con causas operativas.
Para los casos críticos donde se evidencie una mayor presencia de hundimientos de tensión, se pueden poner en práctica mejoras como: instalar descargadores de sobretensión en las líneas de distribución, cables subterráneos, apantallamiento de cables, entre otros.

\section{CONCLUSIONES}

El desarrollo de la metodología le ofrece a CODENSA SA.ESP u otra empresa encargada de la distribución de energía eléctrica, una alternativa que ayuda a identificar y analizar que eventos del sistema se encuentran directamente relacionados con los hundimientos de tensión, con el fin de garantizar un servicio de calidad de energía optimo a todos sus usuarios.

Es de destacar que a partir de los resultados obtenidos en la aplicación de la metodología y la clasificación MSC en las tres subestaciones, se encuentran que el $80 \%$ de los eventos de tipo critico se deben a causa externa (objetos sobre la red, presencia de animales y acciones de terceros), y a su vez se identifica que los hundimientos de tipo severo presentan una mayor concurrencia en cada una de las causas, conllevando a que estos dos tipos de eventos tengan un valor de afectación igual sobre los clientes industriales. Esta metodología proporciona una visión más amplia de la clasificación de las causas que originan los hundimientos de tensión, según normatividad establecida. Actividad que podría conducir a una mejora en la planificación de estrategias, orientadas a resolver muchos de los inconvenientes relacionados con los hundimientos de tensión en las redes de distribución.

Con el desarrollo de la metodología en la etapa 3, los operadores de red podrán identificar y comprobar que los analizadores de red tomen los registros adecuados de los hundimientos de tensión presentados en las barras de distribución, de acuerdo con la normatividad previamente establecida.

De acuerdo a los resultados obtenidos, los eventos ambientales y operativos solo tiene una correlación del 4\% con los hundimientos 
de tensión, lo anterior se puede atribuir a que en el estudio no se tuvieron aspectos como las condiciones del entorno de prestación del servicio (rural-urbano) y el tipo de cargas que manejan los usuarios. Por lo cual se deja abierta la problemática con el fin de determinar qué tipo de causas son las que tiene mayor incidencia en la presencia de hundimientos de tensión.

\section{Referencias Bibliográficas}

[1] M. Vargas; Análisis Registros Calidad de la Potencia Subestación Barzal -EMSA, Universidad Nacional, 2008.

[2] Comisión de Regulación de Energía y Gas (CREG), Resolución 024 de Abril de 2005.

[3] Comisión de Regulación de Energía y Gas (CREG), Resolución 016 de Febrero de 2007.

[4] IEEE Recommended Practice for Monitoring Electric Power Quality. IEEE Std 1159-2009.

[5] J. Sánchez, G. Caicedo, F. Castro; Evaluación de medidores de calidad de potencia eléctrica, Universidad del Valle, 2008.

[6] NTC 5001, Calidad de la potencia eléctrica. Límites y metodología de evaluación en punto de ACOPLE COMUN, 2008.

\section{RECONOCIMIENTOS}

Los autores de este artículo presentan su reconocimiento a la Universidad Nacional de Colombia y al personal de CODENSA S.A ESP, por el apoyo para el desarrollo del estudio acerca de la correlación de sags con eventos del sistema.

[7] Comisión de Regulación de Energía y Gas (CREG), Resolución 097 de Septiembre 2008.

[8] Comisión de Regulación de Energía y Gas (CREG), Resolución 177 de Diciembre de 2009.

[9] G. Cajamarca, M. Romero, A. Pavas, D. Urrutia, L. Gallego, H. Torres, E. Parra; Nueva metodología de análisis comparativo de Sags entre subestaciones de una red de distribución - Caso Colombiano, Simposio Internacional Sobre Calidad De La Energía Eléctrica, Sicel 2007.

[10] IEC 61000-4-30, Standard that defines the correct measuring algorithms for power quality instruments, 2003.

[11] M. Bollen, Understanding power quality problems voltage sags and interruptions, USA 2000, IEEE, 253 - 322. 